\title{
Serum lactate levels are associated with glioma malignancy grade
}

\author{
Mariana Branco $^{\mathrm{a}, \mathrm{b}, *, 1}$, Paulo Linhares ${ }^{\mathrm{b}, \mathrm{c}, 1}$, Bruno Carvalho $^{\mathrm{c}}$, Patrícia Santos ${ }^{\mathrm{d}}$, Bruno M. Costa ${ }^{\mathrm{e}}$, \\ Rui Vaz,c \\ a Serviço de Neurologia, Centro Hospitalar de Entre Douro e Vouga, Portugal \\ ${ }^{\mathrm{b}}$ Faculdade de Medicina da Universidade do Porto, Portugal \\ ${ }^{\mathrm{c}}$ Serviço de Neurocirurgia, Centro Hospitalar de São João, Portugal \\ ${ }^{\mathrm{d}}$ Serviço de Anestesiologia, Centro Hospitalar de São João, Portugal \\ ${ }^{\mathrm{e}}$ Life and Health Sciences Research Institute (ICVS), School of Health Sciences, University of Minho, Portugal
}

\section{A R T I C L E I N F O}

\section{Keywords:}

Glioma

Lactate

Biomarker

Diagnosis

Neuro-oncology

Neurosurgery

\begin{abstract}
A B S T R A C T
Objective: Recent studies have suggested that high grade gliomas are associated with elevated serum lactate concentrations. The aim of the present study is to assess these findings in a sample of patients.

Patients and methods: We reviewed the anesthetic charts of patients with low-grade and high-grade glioma who underwent resection surgery and collected serum lactate concentration before tumor resection, as well as other demographic and tumor-related data (age, gender, WHO grade, and size of the tumor). A statistical comparison between patients with normal $(<2 \mathrm{mmol} / \mathrm{L})$ and elevated $(\geq 2 \mathrm{mmol} / \mathrm{L})$ serum lactate was performed. Results: We included a total of 152 patients (mean age 49.07 years). $62.5 \%$ of patients $(\mathrm{n}=95)$ had a high-grade glioma and $37.5 \%(n=67)$ a low-grade glioma. The multivariate regression showed that high grade gliomas had significantly higher lactate concentration $(\mathrm{p}<0.01$ ). The OR for elevated pre-resection serum lactate increased from 4.94 to 14.33 after adjusting for age and pre-surgical corticosteroid use, and the AUC for the final regression model was 0.98 .

Conclusion: This study reinforces the role of serum lactate as a potential biomarker of brain tumors malignancy, and its results encourage further research on this subject, in order to improve the understanding of this phenomenon and to assess its potential as prognostic and therapeutic monitoring tool.
\end{abstract}

\section{Introduction}

Malignant gliomas are the most common type of primary malignant brain tumors [1]. Glioblastoma(GBM) is a high grade glioma (WHO grade IV) and the most common primary central nervous system(CNS) tumor in European countries and USA [2]. The etiology of GBM is still under debate, but both genetic and epigenetic factors are involved, and glioma stem cells have a major role. The 2016 edition of the World Health Organization (WHO) classification of tumors of the CNS [3] was the first to include guidelines for the use of molecular markers of glioma, indicating that genetic molecular diagnosis and gene analysis of the disease play an increasingly important role [4].

Molecular biomarkers could be an important tool in distinguishing different subtypes of tumors. Therefore, there is a growing need to identify biomarkers of GBM that may have clinical value [5]. The emergence of molecular biomarkers had a significant impact on histological typing and diagnosis, as well as predicting patient survival and response to treatment [6]. However, those biomarkers typically require complex laboratory analyses and are not suitable for pre-operative diagnosis.

Despite the significant progress made in the understanding of oncogenetics and molecular biology of the tumor in recent years, the discovery of therapeutic approaches with significant clinical benefit for GBM patients remains a challenge [4]. In addition to genetic biomarkers, the value of various metabolites as cancer biomarkers have been widely investigated.

Lactate is a final metabolic product of highly proliferative tumors, like GBM, and results from a mechanism of survival of the tumor, which effluxes lactic acid to its microenvironment through transmembrane monocarboxylate transporters (MCTs). According to the conclusions of an in vivo study, inhibition of MCTs function markedly impaired glioma invasion [7]. It is known that hyperlactacidemia may occur in cases of malignancy, specially hematological cancers with liver involvement [8]. We have noticed that a significant number of patients with brain

\footnotetext{
* Corresponding author at: Neurology Department, Centro Hospitalar de Entre o Douro e Vouga, R. Dr. Cândido Pinho 5, 4520-211, Santa Maria da Feira, Portugal. E-mail address: mariana_c_branco@hotmail.com (M. Branco).

${ }^{1}$ These authors contributed equally to this work.
} 
tumors undergoing surgical gross resection or biopsy presented high serum lactate levels ( $\geq 2 \mathrm{mmol} / \mathrm{L}$ ) before or at the beginning of the surgery, without an evident explaining factor. In the majority of cases, the histopathological examination revealed high grade glioma. There is some data supporting a correlation between tumor grade and high presurgical serum lactate level $[9,10]$, therefore we aimed to validate this association in our cohort of patients.

\section{Materials and methods}

\subsection{Study population}

We reviewed the clinical files and anesthetic charts of 152 patients who underwent brain tumor surgery at Hospital São João, a European Union tertiary hospital, from January 2006 to April 2016. We included patients with low-grade and high-grade glioma, who had records of serum lactate concentration obtained by arterial blood gases performed in the operating room after induction of general anesthesia and before skin incision. Additional blood gases were collected during tumor resection but were used only for patient monitoring and were not used for statistical analysis. Patients with known liver or renal dysfunction and with congestive heart failure were excluded, as well as patients who had hemodynamically unstable periods previously to blood gases collection, with necessity of inotropic drugs or blood transfusion $(n=4)$. In addition to serum lactate concentration, we collected demographic, tumor-related data (age, gender, WHO grade, and size of the tumor) and reviewed the clinical files to assess pre-surgery corticosteroid use (in the previous $72 \mathrm{~h}$ ). A pre-resection lactate concentration $\geq 2 \mathrm{mmol} /$ $\mathrm{L}$ was considered as "elevated", according to several studies in literature [11-13]. For tumor volume we performed a diameter-based measurement using MRI imaging and divided them into larger or smaller than $50 \mathrm{~mm}$.

\subsection{Statistical analysis}

Comparisons between patients with normal $(<2 \mathrm{mmol} / \mathrm{L})$ and elevated ( $\geq 2 \mathrm{mmol} / \mathrm{L}$ ) lactate were performed using the Student's $t$-test for independent samples or $\chi 2$ test, where appropriate. The tests were two-sided, with a significance level of 0.05 . To measure associations between tumor grade (high and low grade), serum lactate concentration, and the different clinical and demographic variables we calculated crude and adjusted odds ratio (OR), using univariable and multivariable logistic regression. We fitted all the variables that were significant predictors of tumor grade in a logistic regression model. We assessed the predictive value of an elevated serum concentration to determine tumor grade by estimating the area under the curve of the final model. Statistical analysis was performed using Statistical Package for Social Sciences (SPSS) version 23.

\section{Results}

We included a total of 152 patients, 88 males and 64 females. The mean age was 49.07 (standard deviation(SD) $=19.72$ years), $62.5 \%$ of patients $(n=95)$ had a high-grade glioma and $37.5 \%(n=67)$ a lowgrade (Table 1). Patients with high-grade glioma were on average older than patients with low-grade glioma (58.6 vs. 33.12 years old; $\mathrm{p}<0.01$ ). In low-grade gliomas, the mean lactate concentration was $1.38 \mathrm{mmol} / \mathrm{L}(\mathrm{SD}=0.61)$, and in high-grade gliomas it was 2.03 $(\mathrm{SD}=0.81)$.

There were $42.1 \%$ patients with elevated pre-resection serum lactate $(\mathrm{n}=64)$, and the mean lactate level was $1.79 \mathrm{mmol} / \mathrm{L}$. When comparing patients divided according to their serum lactate (normal $(<2 \mathrm{mmol} / \mathrm{L})$ versus elevated $(\geq 2 \mathrm{mmol} / \mathrm{L}))$ we found significant associations between higher serum lactate and higher tumor grade and female gender $(\mathrm{p}<0.01)$, and no significant associations with age or tumor size (Table 2). There was a significant association between pre-
Table 1

Demographics and clinical characteristics of the study patients.

\begin{tabular}{ll} 
Gender, $\%$ & \\
$\quad$ Male & $57.9 \%$ \\
$\quad$ Female & $42.1 \%$ \\
Age, years (mean, SD) & $49.07(19.72)$ \\
Tumor grade, $\%$ & \\
$\quad$ High & $62.5 \%$ \\
$\quad$ Low & $37.5 \%$ \\
Tumor size & \\
$\quad \geq 50 \mathrm{~mm}$ & $34.9 \%$ \\
$\quad<50 \mathrm{~mm}$ & $48.0 \%$ \\
Serum lactate, $\%$ & \\
$\quad$ Elevated & $42.1 \%$ \\
$\quad$ Normal & $57.9 \%$ \\
Serum lactate concentration, mmol/L (mean, SD) & $1.79(0.81)$ \\
& \\
\hline
\end{tabular}

Table 2

Comparison of patients with high and low pre-resection serum lactate concentrations.

\begin{tabular}{llll}
\hline Variable & $\begin{array}{l}\text { High lactate } \\
(\geq 2 \mathrm{mmol} / \mathrm{L})\end{array}$ & $\begin{array}{l}\text { Normal lactate } \\
(<2 \mathrm{mmol} / \mathrm{L})\end{array}$ & p-value \\
\hline $\begin{array}{l}\text { Tumor grade, } \% \\
\quad \text { High }\end{array}$ & $56.8 \%$ & & \\
$\quad$ Low & $21.1 \%$ & $73.2 \%$ & $<0.01$ \\
$\quad$ Gender, $\%$ & & $78.9 \%$ & \\
$\quad$ Male & $33.0 \%$ & $67.0 \%$ & $<0.01$ \\
$\quad$ Female & $57.8 \%$ & $42.2 \%$ & 0.34 \\
Age (mean, SD) & $50.8(18.7)$ & $47.7(20.5)$ & \\
Tumor size, $\%$ & & & 0.27 \\
$\quad \geq 50 \mathrm{~mm}$ & $47.4 \%$ & $37.7 \%$ & \\
$\quad<50 \mathrm{~mm}$ & $52.6 \%$ & $62.3 \%$ & \\
\hline
\end{tabular}

SD - standard deviation.

surgery corticosteroid use and lactate levels, with a mean lactate level of $1.61 \mathrm{mmol} / \mathrm{L}$ in patients with corticosteroid use vs. $1.89 \mathrm{mmol} / \mathrm{L}$ in patients with no registered use of these drugs $(p=0.04)$

In the univariate logistic regression, there was a significant association between elevated pre-resection serum lactate and high tumor grade $(\mathrm{OR}=4.94 ; \mathrm{p}<0.01$; AUC 0.70). A high tumor grade was also associated with older age $(\mathrm{OR}=1.10 ; \mathrm{p}<0.01)$ and increased presurgery corticosteroid use ( $\mathrm{OR}=2.61 ; \mathrm{p}=0.02)$. There was no association between tumor size and sex with tumor grade (Table 3 ).

In the multivariate regression model, both older age, corticosteroid use, and elevated lactate retained their association with high tumor grade (Table 3), implying that, after adjusting for all other factors, increased lactate concentration are a predictor of high-grade gliomas. The OR for elevated pre-resection serum lactate increased from 4.94 to 14.33 after adjusting for age and corticosteroid, and the AUC for the final regression model was 0.98 .

Table 3

Logistic regression analysis of the association between tumor grade and preresection serum lactate concentrations.

\begin{tabular}{|c|c|c|c|c|}
\hline & \multicolumn{2}{|c|}{ Univariate logistic regression } & \multicolumn{2}{|c|}{ Logistic regression model } \\
\hline & $\begin{array}{l}\text { Odds Ratio (95\% } \\
\text { CI) }\end{array}$ & p-value & $\begin{array}{l}\text { Odds Ratio } \\
(95 \% \mathrm{CI})\end{array}$ & p-value \\
\hline $\begin{array}{l}\text { Lactacte level } \\
\qquad(\geq 2 \mathrm{mmol} / \mathrm{L})\end{array}$ & $\begin{array}{l}4.94 \\
(2.32-10.51)\end{array}$ & $<0.01$ & $\begin{array}{l}14.33 \\
(4.01-56.07)\end{array}$ & $<0.01$ \\
\hline Age, years & $\begin{array}{l}1.10 \\
(1.06-1.13)\end{array}$ & $<0.01$ & $\begin{array}{l}1.13 \\
(1.08-1.17)\end{array}$ & $<0.01$ \\
\hline Corticosteroid use & $\begin{array}{l}2.61 \\
(1.31-5.17)\end{array}$ & 0.02 & $\begin{array}{l}9.49 \\
(2.86-31.45)\end{array}$ & $<0.01$ \\
\hline Sex, male & $\begin{array}{l}1.59 \\
(0.83-3.07)\end{array}$ & 0.16 & & \\
\hline $\begin{array}{l}\text { Tumor size } \\
\quad \geq 50 \mathrm{~mm}\end{array}$ & $\begin{array}{l}1.19 \\
(0.58-2.44)\end{array}$ & 0.63 & & \\
\hline
\end{tabular}




\section{Discussion}

Increased glucose uptake is a common feature of malignant tumor cells, with consequent production of lactic acid, even in aerobic conditions, and it is known that a lactate accumulation occurs in the GBM microenvironment $[7,14]$. Kohli-Seth et al provided the first evidence of the systemic repercussion of this phenomenon in a retrospective study reporting hyperlactacidemia in a large number of patients from an intensive care unit (ICU) submitted to brain tumor resection surgery, but they did not correlate these findings with tumor pathology [15]. In 2016, Kahlon et al. published a case of chronically elevated lactate levels in a patient with GBM as a circumstance not previously reported, revealing an unawareness of the scientific community to the serum lactate behavior in this type of cancer [16].

Two previous studies have aimed to find an association between serum lactate and gliomas malignancy $[9,10]$. Mariappan et al observed the pre-surgical lactate levels of 50 patients undergoing brain surgery for glioma resection and found an association between high lactate levels and high-grade tumors [9]. Shih C. et al, aimed to identify potential pretreatment serum biomarkers that might influence oncological outcomes in patients with primary brain tumor and found the same association [10].

In the present study, we found a significant association between high grade glioma and high levels of pre-surgical serum lactate. There is not a clear definition of the values from which serum lactate concentration is considered to be elevated, but most studies use cut-offs between 2.0 and $2.5 \mathrm{mmol} / \mathrm{L}$ [8]. Therefore, we defined values equal or above $2.0 \mathrm{mmol} / \mathrm{L}$ as "elevated". Our findings confirmed the results described in previous studies, but in a larger sample and assessing the effect of potential confounding factors not previously evaluated, which reinforces the strength of this hypothesis. However, we did not evaluate serum lactate levels at other phases or after surgery. Furthermore, we did not assess the role of serum lactate levels as a predictor of survival. A previous study by Cata et al. found no association between lactate levels and survival in gliomas, suggesting that serum lactate has probably more potential as a biomarker for diagnostic and staging than prognostic [17].

We excluded patients with known liver or renal dysfunction and with congestive heart failure, because these conditions are known to cause a rise in serum lactate. High-dose corticosteroid therapy could also potentially rise lactate levels [18] and a large number of patients with high grade glioma are under this type of therapy. Previous studies did not assess this factor. We reviewed the clinical files of our patients in order to determine if they underwent corticosteroid therapy before surgery and found that the effect of high lactate concentration in predicting high-grade gliomas is not changed after adjusting for the use of corticosteroid in the multivariable model. This finding suggests that the observed increase in mean lactate levels in patients with high grade gliomas is related to the tumor biology, and not to the use of corticosteroids. Nevertheless, we have to be careful in establishing a definitive conclusion. Given the retrospective nature of this review, we cannot exclude that information on corticosteroid therapy could be absent in some of the clinical files, particularly pre-admission use. Future prospective studies are warranted to indisputably resolve this issue, namely by systematically and per study protocol measuring lactate levels before any corticosteroid use in a consecutive group of patients.

These results are encouraging in what concerns to the usefulness of serum lactate determination in the diagnosis of gliomas, and they raise some additional questions and hypothesis that should be addressed in future studies. It would be interesting to compare serum lactate levels with MRI spectroscopy results, since it has been showed that malignant transformation of the glial tumors is accompanied by the presence of elevated lactate peaks in MR spectroscopy of high grad gliomas [19]. Indeed, an association between elevated lactate peak in MR spectroscopy and hyperlactacidemia in high-grade gliomas would support the role of the tumor as the cause of the metabolic phenomenon leading to hyperlactatemia and, consequently, the potential of serum lactate as a biomarker of glioma malignancy. A panel of biomarkers for tumor malignancy, including lactate, could be probably more helpful than any single isolated measurement. Lactate-related enzymes, such as lactate dehydrogenase (LDH) are particularly promising. It is known that LDHA, which catalyses the interconversion of pyruvate and lactate, is upregulated in human cancers, including GBM [20]. Lutterbach et al. showed that raised serum LDH levels may negatively influence outcome in glioblastoma patients [21]. This findings suggest that LDH could be an interesting biomarker of glioma grade, Although LDH may be elevated in several situations, like hemolysis, thromboembolism, systemic infection and inflammation and muscle injury [22], it could potentially represent an additional and perhaps more sensitive tool than serum lactate in the diagnosis of high grade glioma. Additional studies are also warranted to determine if this method could also be suitable for the differential diagnosis between gliomas and other brain tumors, like metastasis and lymphomas

\section{Conclusion}

In all, this study reinforces the role of serum lactate as a potential biomarker of brain tumors malignancy, and its results encourage further research on this subject.

\section{Ethical statement}

All procedures performed in studies involving human participants were in accordance with the ethical standards of the institutional research committee and with the 1964 Helsinki declaration and its later amendments or comparable ethical standards.

\section{Declaration of Competing Interest}

The authors declare that they have no conflict of interest.

\section{References}

[1] A. Omuro, L.M. DeAngelis, Glioblastoma and other malignant gliomas: a clinical review, JAMA 310 (17) (2013) 1842-1850.

[2] C. Adamson, O.O. Kanu, A.I. Mehta, et al., Glioblastoma multiforme: a review of where we have been and where we are going, Expert Opin. Invest. Drugs 18 (8) (2009) 1061-1083.

[3] D.N.O.H. Louis, O.D. Wiestler, W.K. Cavenee, WHO Classification of Tumours of the Central Nervous System -Revision, 4th edition, WHO, 2016.

[4] L. Zhou, H. Tang, F. Wang, et al., Bioinformatics analyses of significant genes, related pathways and candidate prognostic biomarkers in glioblastoma, Mol. Med. Rep. 18 (5) (2018) 4185-4196.

[5] E.T. Sayegh, G. Kaur, O. Bloch, A.T. Parsa, Systematic review of protein biomarkers of invasive behavior in glioblastoma, Mol. Neurobiol. 49 (3) (2014) 1212-1244.

[6] A. Mansouri, L.D. Hachem, S. Mansouri, et al., MGMT promoter methylation status testing to guide therapy for glioblastoma: refining the approach based on emerging evidence and current challenges, Neuro Oncol. (2018).

[7] C.B. Colen, Y. Shen, F. Ghoddoussi, et al., Metabolic targeting of lactate efflux by malignant glioma inhibits invasiveness and induces necrosis: an in vivo study, Neoplasia 13 (7) (2011) 620-632.

[8] L.W. Andersen, J. Mackenhauer, J.C. Roberts, K.M. Berg, M.N. Cocchi, M.W. Donnino, Etiology and therapeutic approach to elevated lactate levels, Mayo Clin. Proc. 88 (10) (2013) 1127-1140.

[9] R. Mariappan, L. Venkatraghavan, A. Vertanian, et al., Serum lactate as a potential biomarker of malignancy in primary adult brain tumours, J. Clin. Neurosci. 22 (1) (2015) 144-148.

[10] C.C. Shih, T.S. Lee, F.Y. Tsuang, et al., Pretreatment serum lactate level as a prognostic biomarker in patients undergoing supratentorial primary brain tumor resection, Oncotarget 8 (38) (2017) 63715-63723.

[11] S. Bharadwaj, L. Venkatraghavan, R. Mariappan, et al., Serum lactate as a potential biomarker of non-glial brain tumors, J. Clin. Neurosci. 22 (10) (2015) 1625-1627.

[12] R. Mariappan, L. Venkatraghavan, A. Vertanian, et al., Serum lactate as a potential biomarker of malignancy in primary adult brain tumours, J. Clin. Neurosci. 22 (1) (2015) 144-148.

[13] A.L. Shetty, K. Thompson, K. Byth, et al., Serum lactate cut-offs as a risk stratification tool for in-hospital adverse outcomes in emergency department patients screened for suspected sepsis, BMJ Open 8 (1) (2018) e015492.

[14] J. Li, S. Zhu, J. Tong, et al., Suppression of lactate dehydrogenase A compromises 
tumor progression by downregulation of the Warburg effect in glioblastoma, Neuroreport 27 (2) (2016) 110-115.

[15] R. Kohli-Seth, S.R. Mukkera, A.B. Leibowitz, et al., Frequency and outcomes of hyperlactatemia after neurosurgery: a retrospective analysis, ICU Dir. 2 (6) (2011) 211-214.

[16] A.S. Kahlon, M. Alexander, A. Kahlon, J. Wright, Lactate levels with glioblastoma multiforme, Proceedings (Baylor University Medical Center) 29 (3) (2016) 313-314.

[17] J.P. Cata, S. Bhavsar, K.B. Hagan, et al., Intraoperative serum lactate is not a predictor of survival after glioblastoma surgery, J. Clin. Neurosci. 43 (2017) 224-228.

[18] T.H. Ottens, M.W.N. Nijsten, J. Hofland, et al., Effect of high-dose dexamethasone on perioperative lactate levels and glucose control: a randomized controlled trial,
Crit. Care (London, England) 19 (1) (2015) 41-41.

[19] M. Bulik, R. Jancalek, J. Vanicek, A. Skoch, M. Mechl, Potential of MR spectroscopy for assessment of glioma grading, Clin. Neurol. Neurosurg. 115 (2) (2013) 146-153.

[20] S. Daniele, C. Giacomelli, E. Zappelli, et al., Lactate dehydrogenase-A inhibition induces human glioblastoma multiforme stem cell differentiation and death, Sci. Rep. 5 (2015) 15556.

[21] J. Lutterbach, W. Sauerbrei, R. Guttenberger, Multivariate analysis of prognostic factors in patients with glioblastoma, Strahlenther. Onkol. 179 (1) (2003) 8-15.

[22] C. Perry, H. Peretz, O. Ben-Tal, A. Eldor, Highly elevated lactate dehydrogenase level in a healthy individual: a case of macro-LDH, Am. J. Hematol. 55 (1) (1997) $39-40$. 